\title{
Nueva propuesta de acceso a la universidad en España, ¿una herramienta para erradicar las desigualdades sociales?
}

\author{
Revesado Carballares, David \\ Facultad de Educación- Universidad de Salamanca, Salamanca, España \\ drevesado@usal.es \\ García Redondo, Eva \\ Facultad de Educación- Universidad de Salamanca, Salamanca, España \\ evagr@usal.es \\ González López, Víctor \\ Facultad de Educación- Universidad de Salamanca, Salamanca, España \\ vgl@usal.es
}

\section{Resumen}

Durante los últimos años, el sistema educativo español viene asistiendo a una profunda transformación, fruto, entre otras cosas, de la alternancia política instaurada en nuestro país, que ha traído consigo la promulgación de numerosas reformas educativas, en un periodo de tiempo muy breve. La última de ellas pretende llevar a cabo una serie de cambios que afectan a diferentes ámbitos de nuestro sistema, entre ellos, el acceso universitario. En muchas ocasiones, este se ve condicionado por diferentes factores que giran en torno al alumno, y que van más allá de lo estrictamente académico, como son: el origen socioeconómico familiar, el nivel educativo de los progenitores, el centro de enseñanza, etc. El largo y sinuoso camino que los alumnos deben recorrer hasta que comienzan su periplo universitario está determinado por una gran cantidad de elementos sobre los que se pueden esconder serias desigualdades sociales.

Valiéndonos del enfoque sociohistórico, pretendemos analizar la nueva propuesta educativa en materia de acceso, a partir de la cual, se dotará de una mayor autonomía a las instituciones universitarias, a fin de que sean ellas mismas las encargadas de seleccionar a sus alumnos, a través de pruebas específicas de acceso, tal y como ya hacen las principales potencias educativas del panorama internacional. De este modo, se producirá un cambio de paradigma en el acceso universitario, en donde las instituciones de educación superior podrán seleccionar a sus alumnos atendiendo a sus credenciales, obviando cualquier otro elemento que se aleje de lo académico y que pueda traer consigo desigualdades en este proceso de transición.

\section{Abstract}

During the last few years, the Spanish educational system has assisted a profound transformation, produce, among other things, of the political alternation established in our country, which has brought with him the enactment of numerous educational reforms, in a very short period of time. The last pretend to carry out a series of changes that affect different areas of our system, including university access. Many times, this is conditioned by different factors that revolve around the student, and that go beyond the strictly academic, such as: the family socioeconomic origin, the educational level of the parents, the teaching center, etc. The long and sinuous road that students must go over until they begin their university journey is determined by a lot of elements on which serious social inequalities can be hidden.

Using the sociohistorical approach, we intend to analyze the new educational proposal in terms of access, from which, university institutions will be given greater autonomy, so that they themselves are in charge of selecting their students, through of specific access tests, as the main educational powers of the international panorama already do. In this way, there will be a paradigm shift in university access, where higher education institutions will be able to select their students according to their credential, obviating any other element that moves away from academics and that may bring about inequalities in this transition process.

Palabras clave: Reforma educativa, Acceso, Universidad, España, Igualdad de oportunidades.

Keywords: Educational reform, Access, University, Spain, Equal opportunities. 


\section{INTRODUCCIÓN}

A lo largo de las últimas décadas, se ha producido un constante crecimiento en la demanda de educación superior ${ }^{1}$ en nuestra sociedad, la denominada Sociedad del Conocimiento, y se prevé que sea aún mayor a lo largo de los próximos años, llegando a superar las previsiones y de los sistemas educativos (Muñoz Vitoria, 1993). Frente a tal contexto, los centros de educación superior están destinados a desempeñar un papel fundamental, donde los esquemas clásicos de producción, difusión y aplicación del saber experimentarán grandes cambios (UNESCO, 2005).

Si examinamos de una manera genérica el contexto de la educación superior, podemos afirmar que esta estaría compuesta por tres etapas o momentos clave: acceso, retención o formación y empleabilidad (European Commission, 2015). Nuestra intención a lo largo de este trabajo es la de examinar, brevemente, esta primera etapa del continuum que conforma el aparato universitario en nuestro país. Sin duda alguna, la temática que aquí presentamos está adquiriendo una gran relevancia en los últimos tiempos, no solo en el contexto nacional, sino también en el plano internacional; los procesos de transición y, especialmente, el que se refiere de la Educación Secundaria hacia la Educación Superior está tomando un papel muy importante, generando un gran atractivo para los investigadores educativos, especialmente en perspectiva internacional o comparada.

Son muchos los estudios publicados a lo largo de los últimos años, que defienden que el acceso hacia la Educación Superior está condicionado por numerosos elementos que van más allá de lo estrictamente académico, y que pueden generar serias desigualdades en este complejo proceso de transición, como son: el origen social del educando (Ariño y Llopis, 2011; Barañano y Finkel, 2014; Dupriez, Monseur, Van Campenhoudt y Lafontaine, 2010; Enciso, 2013), la renta económica familiar del alumno (Bernardi y Cebolla, 2014; Fernández, 2008; Rogero-García y Andrés- Candelas, 2014; Villar y Hernández, 2015), los centros de secundaria al que los alumnos están adscritos (Moreno, Sánchez y Jiménez, 2015), etc. Es por ello por lo que, a lo largo de este trabajo, vamos a analizar la nueva propuesta de acceso hacia la universidad en nuestro país, a partir de la Ley Orgánica para la Mejora de la Calidad Educativa (LOMCE), promulgada el pasado 2013, de la mano del Partido Popular (PP). Esta, pretende transformar el modelo de acceso universitario, pasando de un modelo abierto, carente de restricciones, a un modelo cerrado, en donde se valorará la adecuación del alumno a los estudios elegidos, mediante mecanismos selectivos de ingreso. De esta forma, se dejará en un segundo plano otros elementos que puedan tener una cierta incidencia en este proceso de transición, y que se alejan de lo estrictamente académico, tal y como son: el origen socioeconómico del alumno, los centros de educación secundaria en los que el alumno está matriculado, nivel cultural de los progenitores, etc.

\section{NUEVA PROPUESTA DE ACCESO: DOTAR DE UNA MAYOR AUTONOMÍA A LAS INSTITUCIONES UNIVERSITARIAS}

El acceso a la universidad española ha sufrido numerosas modificaciones a lo largo de las últimas cuatro décadas, fruto de la gran alteración política instaurada en nuestro país, que ha traído consigo la promulgación de numerosas leyes educativas, en un periodo de tiempo muy breve. Si bien es cierto que estos cambios no han modificado sustancialmente el modelo de acceso ya que, hasta la reciente puesta en marcha de la LOMCE, estos eran muy superfluos, y no llevaban a cabo transformaciones de gran envergadura.

En este sentido, la nueva ley propone suprimir la Prueba de Acceso a la Universidad, conocida coloquialmente como "Selectividad», y que tiene sus raíces en la etapa tecnocrática del franquismo. Esta medida no es nueva entre nuestras fronteras ya que, en el pasado, existieron varios intentos para suprimirla, aunque no tuvieron demasiado éxito. La primera de ellas, con la llegada de

\footnotetext{
${ }^{1}$ Valiéndonos de la Clasificación Internacional Normalizada de la Educación (CINE), elaborada por la UNESCO (2013), utilizaremos esta terminología a lo largo de nuestro trabajo para hacer referencia a los programas de educación terciaria de ciclo corto (CINE 5) y los grados en educación terciaria o equivalentes (CINE 6).
} 
la democracia, cuando se propuso la derogación de esta prueba con el fin de otorgar una mayor autonomía a las instituciones universitarias. Y, en segundo lugar, de una forma más reciente, en el año 2003, con el Partido Popular al frente, también se propuso la abolición de la selectividad, mediante la Ley Orgánica de Calidad de la Educación (LOCE); finalmente, la llegada al poder del Partido Socialista Obrero Español (PSOE) un año más tarde, trunco de lleno la posibilidad de llevar a cabo esta propuesta.

Por otro lado, se pretende reinstaurar la reválida. Esta prueba, que se llevará a cabo en los propios centros de Educación Secundaria, deberá ser superada por todos los alumnos para la obtención del título de bachiller, un título que, desde este momento, pasará a ser el único requisito indispensable para el acceso universitario. No obstante, para complementar esta segunda medida, se pretende llevar a cabo una tercera que, sin duda alguna, será determinante para la conformación de un nuevo modelo de acceso universitario. Esta consiste en dotar con una mayor autonomía a las facultades universitarias, permitiendo que sean ellas mismas las encargadas de seleccionar a sus alumnos, mediante aquellos procedimientos que crean más adecuados:

Además, las Universidades podrán fijar procedimientos de admisión a las enseñanzas universitarias oficiales de grado de alumnos y alumnas que hayan obtenido el título de Bachiller o equivalente, de acuerdo con la normativa básica que establezca el Gobierno, que deberá respetar los principios de igualdad, no discriminación, mérito y capacidad (Artículo 38.2, Ley Orgánica 8/2013, para la Mejora de la Calidad Educativa).

\section{TRANSFORMANDO EL MODELO DE ACCESO A LA UNIVERSIDAD ESPAÑOLA}

El acceso al sistema de Educación Superior, como es lógico, no se lleva a cabo de una forma homogénea en el contexto internacional, dado que cada sistema educativo posee unas características diferenciales del resto, que dificultan este proceso. Con las declaraciones de La Sorbona (1998) y Bolonia (1999), se puso en marcha el, ya conocido por todos, Espacio Europeo de Educación Superior (EEES), que tiene como objetivo crear un área educativa común de Enseñanza Superior que abarque toda la Comunidad Europea (Valle, 2001). Sin embargo, pese a que el EEES ha hecho hincapié en la armonización de numerosos aspectos del sistema universitario, como su estructura (el famoso 3+2), la implantación de un sistema de créditos ECTS, nuevos métodos de aprendizaje y medios técnicos, etc. lo cierto es que algo tan relevante como es el acceso, no ha sido abordado. Es por ello, por lo que, a continuación, creemos conveniente definir y analizar los distintos modelos de acceso hacia tales estudios.

\subsection{Modelos de acceso a la educación superior}

A la hora de hablar de los diferentes modelos que dan acceso al sistema universitario, debemos hacer mención, especialmente, a tres de ellos: modelo abierto, modelo cerrado y modelo entreabierto. Sin duda alguna, estos gozan de una gran importancia en este proceso de transición que estamos analizando, puesto que cada uno de ellos tiene unas características específicas que pueden traer consigo consecuencias muy distintas.

El primero de ellos, el modelo abierto, permitirá que todos los alumnos que estén capacitados para seguir con los estudios universitarios lo hagan; se trata, de un modelo totalmente accesible, donde no existe ningún tipo de restricción. El principal problema que presenta este modelo es, sin duda alguna, el gran desajuste provocado entre número de titulados y las necesidades que presenta el sistema económico productivo, dado que el número de titulados será muy elevado, y no todos tendrán cabida dentro del mercado laboral (Valle, 2001). Como consecuencia de ello, podemos encontrarnos con problemas como la sobrecualificación, fuga de cerebros o brain drain, etc. por no hablar de importantes pérdidas económicas que supone formar a personas que, posteriormente, no van a poder desarrollarse desde el punto de vista profesional.

Por otro lado, haremos referencia al modelo cerrado, en donde no todos aquellos alumnos que estén capacitados para seguir los estudios universitarios podrán hacerlo, ya que se producirá 
una selección rigurosa de los candidatos, con el fin de determinar que el número de alumnos que accedan al sistema universitario sea similar a la demanda del mercado laboral. Este modelo presenta, como principal ventaja, el gran ajuste producido sobre el sistema económico productivo, ya que el número de titulados se acordará según las necesidades del mercado laboral. Por el contrario, el gran problema que presenta es que, en cierta medida, vulnera uno de los pilares del estado español, como es el derecho a la educación, dado que el acceso estará altamente restringido (Valle, 2001).

Por último, hablaremos del modelo entreabierto, que limita el acceso universitario, pero no de una forma tan rigurosa como para pensar exclusivamente en las necesidades del mercado laboral. Este modelo, por tanto, presentará una cierta accesibilidad, ya que esta limitación de la que hablamos se llevará a cabo tan solo en aquellas titulaciones donde la demanda sea muy elevada o, por el contrario, donde la oferta académica sea más restringida. Por tanto, podríamos afirmar, que este modelo sería el más adecuado, ya que pretende aunar los beneficios de los dos sistemas anteriormente descritos: derecho a la educación y ajuste entre oferta de titulados y la demanda del mercado laboral (Valle, 2001).

\subsection{Nuevo modelo de acceso a la universidad española}

La triple propuesta llevada a cabo por la LOMCE, que hemos descrito en el primero apartado de nuestro trabajo está, encaminada hacia la transformación del modelo de acceso al sistema universitario español, un modelo que, como ya hemos señalado, lleva vigente en nuestro país desde finales del franquismo. De consolidarse esta propuesta, pasaremos de un modelo de acceso abierto, carente de restricciones, más allá de la superación de una prueba que muchos autores catalogan como, meramente, cultural (Vega y Hernández, 2015), a un modelo de acceso cerrado, donde las instituciones universitarias gozarán de autonomía para seleccionar a sus alumnos atendiendo a aquellos criterios que crean más convenientes.

\section{DISCUSIÓN Y CONCLUSIONES}

Pese a los constantes cambios políticos y numerosas reformas educativas que se han llevado a cabo a lo largo de los últimos tiempos, la realidad nos muestra que, el acceso a la universidad en España ha sido y sigue siendo el mismo que el que se instauró en nuestro país hace casi medio siglo. De este modo, este proceso de transición que aquí analizamos sigue supeditado a la superación de una prueba de carácter cultural que, por otro lado, poco a poco, va adquiriendo tintes competenciales (Vega, 2017). Pese a que la PAU ha sufrido pequeñas modificaciones en cuanto a su denominación, e incluso en cuanto a su estructura, lo cierto es que mantiene la misma esencia que hace casi cincuenta años. Sin embargo, los cambios que trae consigo la LOMCE en materia de acceso pretenden transformar este modelo arcaico y fuertemente arraigado al tradicionalismo.

De concretarse la nueva propuesta (recordemos que hasta la fecha no lo ha hecho ya que, desde la promulgación de la nueva ley educativa, se ha regulado este proceso de transición de forma provisional, a través de diferentes órdenes ministeriales y regionales que son aprobadas año a año), se producirá un cambio de paradigma en el acceso universitario español, en donde las instituciones de Educación Superior gozarán de autonomía para seleccionar a sus alumnos, atendiendo a sus credenciales, y obviando, de este modo, cualquier otro elemento que se aleje de lo estrictamente académico y que pueda traer consigo desigualdades sobre este proceso de transición. Sin embargo, es importante tener en cuenta que para que esto ocurra, será necesario llevar a cabo un cambio que vaya más allá de lo institucional, y se centre en lo político, siguiendo aquellas medidas que dictan los textos normativos; de no ser así, nunca conseguiremos que estas propuestas educativas acaben de consolidarse.

Por lo tanto, esta autonomía universitaria a la que venimos aludiendo a lo largo de nuestro trabajo, tiene como objetivo regular este proceso de transición de una forma más justa y equitativa, valoran- 
do las credenciales académicas de los alumnos para, de este modo, podamos asegurar la idoneidad de los mismos. De este modo, se restará incidencia a aquellos elementos que se alejen de lo académico y que, sin duda alguna, en la actualidad aún están muy presentes sobre este proceso de transición, como son factores económicos, sociales, culturales, etc.

\section{REFERENCIAS BIBLIOGRÁFICAS}

Ariño, A. y Llopis, R. (2011). ¿Universidad sin clases? Condiciones de vida de los estudiantes universitarios en España (Eurostudent IV). Madrid: Ministerio de Educación.

Barañano, M. y Finkel, L. (2014). Transmisión intergeneracional y composición social de la población estudiantil universitaria española: cambios y continuidades. Revista de asociación de Sociología de la Educación, 7 (1), pp. 42-60.

Bernardi, F. y Cebolla, H. (2014). Clase social de origen y rendimiento escolar como predictores de las trayectorias educativas. Revista Española de Investigaciones Sociológicas, 146, pp. 3-22.

Dupriez, V., Monseur, C., Van Campenhoudt, M. y Lafontaine, D. (2012). Social inequalities of post-secondary educational aspirations: influence of social background, school composition and institutional context. European Educational Research Journal, 11(4), pp. 504-519.

Enciso, M. I. (2013). Origen social de los graduados y la equidad en el acceso a la universidad. Revista de educación superior, 165, vol. 42, pp. 11-29.

European Commission/EACEA/Eurydice. (2015): Modernisation of Higher Education in Europe: Access, Retention and Employability 2014, Eurydice Report (Luxembourg: Publications Office of the European Union).

Fernández, M. (2008). Escuela pública y privada en España: La segregación rampante. Revista de la Asociación de Sociología de la Educación, 1(2), pp.42-69.

Moreno, D., Sánchez, J. y Jiménez, J. D. (2014). ¿En los centros privados se inflan las calificaciones de los estudiantes? Revista de Educación, 366, pp. 243-266.

Muñoz Vitoria, F. (1993). El sistema de acceso a la universidad en España 1940-1990. Madrid: CIDE.

Rogero-García, J. y Andrés-Candelas, M. (2014). Gasto público y de las familias en educación en España: diferencias entre centros públicos y concertados. Revista Española de Investigaciones Sociológicas, 147, pp. 121-132.

UNESCO. (2005). Hacia las sociedades del conocimiento. Paris: Ediciones Unesco.

UNESCO. (2013): Clasificación Internacional Normalizada de la Educación (Montreal: Instituto de Estadística de la UNESCO).

Valle, J. M. (2001): Desde la secundaria a la universidad: tentativa de modelos para un problema poliédrico, Revista Española de Educación Comparada, 7, pp. 191-227.

Valle, J. M. (2008): Hacia el pasaporte europeo universitario: Armonización de los sistemas de acceso a la universidad. En J. L. García (Ed.), Formar ciudadanos europeos, pp. 99-156 (Madrid: Academia Europea de Ciencias y Artes).

Vega, L. (2017). La globalización en los procesos y programas de formación de maestros en España. Qué podemos aprender y qué debemos mejorar. Hacia una reconsideración del modelo de formación. En M.V. Pires; C. Mesquita; R.P. Lopes; G. Santos; M. Cardoso; J. S. de P. C. Sousa y C. Teixeira (Eds.), Livro de atas do II Encontro Internacional de Formação na Docencia (INCTE) (pp. 27-38). Bragança: Instituto Politécnico de Bragança. 
Vega, L. y Hernández, J. C. (2015). Spain and France: Moving from democratization towards elitism in access to higher education. EN: V. STEAD (ED.), International Perspective on Higher Education Admission Policy. A Reader. New York: Peter Lang.

Villar, A. y Hernández, F. J. (2015). ¿Las familias con mayor poder adquisitivo resultan beneficiadas de la inversión pública educativa? ARXIUS, 32, pp. 161-172. 